\title{
Le retour des poissons migrateurs
}

\author{
J.M. GOUGIS et P. STEINBACH \\ Conseil Supérieur de la Pêche
}

\section{LES POISSONS MIGRATEURS : UN PATRIMOINE IRREMPLAÇABLE}

Outre la valeur patrimoniale de chaque espèce, les poissons migrateurs sont des indicateurs de la qualité d'ensemble des cours d'eau et de leurs particularités. Ils témoignent de l'état fonctionnel de l'hydrosystème et méritent, à ce titre, qu'on leur accorde une attention toute particulière.

En effet, chaque bassin possède son propre peuplement de poissons migrateurs. Cette identification hydrographique est forgée, de génération en génération, par le retour des adultes en rivière sur leur lieu de naissance, excepté pour l'anguille qui se reproduit en mer.

Au plan de la gestion, l'existence et les besoins de ce patrimoine biologique cadrent parfaitement avec l'approche de bassin qui s'impose aux gestionnaires depuis la loi sur l'eau du 3 janvier 1992.

Hormis l'anguille et la truite de mer, tous les grands migrateurs figurent à l'annexe II de la directive 92/43/CEE portant sur la conservation des habitats naturels ainsi que de la flore et de la faune sauvages : l'esturgeon, le saumon atlantique, les deux aloses (Alosa alosa et Alosa fallax) et les deux lamproies (Petromizon marinus et Lampetra fluviatilis).

Il s'agit d'espèces d'intérêt communautaire dont les habitats sont à conserver en raison de leurs statuts d'espèces endémiques et vulnérables.

L'esturgeon, seule espèce piscicole prioritaire (figurant à l'annexe IV de la directive) a disparu du bassin de la Loire vers 1940. La souche de saumon qui subsiste sur l'axe Loire-Allier a une valeur patrimoniale qui dépasse largement le territoire ligérien. Cette population de grands saumons correspond à la dernière souche d'Europe occidentale possédant les caractéristiques biologiques nécessaires à la colonisation des grands bassins fluviaux.

Les poissons migrateurs constituent également une ressource économique non négligeable. L'essentiel du chiffre d'affaire des pêcheurs professionnels en activité dans l'estuaire et le bassin de la Loire (plus de $30 \mathrm{MF} / \mathrm{an}$ ) repose sur les espèces migratrices, en particulier sur celles qui restent les plus abondantes : l'anguille et la lamproie. En outre, la pêche de loisirs offre un important potentiel de mise en valeur économique à travers les activités directement ou indirectement liées à la pêche des poissons tels que le saumon.

\section{I 圆 LA RICHESSE DE CHAQUE MIGRATEUR}

\subsection{Le Saumon}

La phase de développement des jeunes saumons dans le Haut-Allier est caractérisée par un mode de croissance élevé permettant une smoltification et une dévalaison précoces, dès l'âge de 1 an chez près de $75 \%$ des tacons.

Cette prédominance d'individus à croissance rapide est courante sous nos latitudes. Elle résulte des conditions de milieu, notamment du régime thermique des rivières françaises, favorable au développement de juvéniles.

Par contre la durée de séjour du Saumon de Loire sur ses aires de grossissement en mer est une caractéristique unique que l'on ne retrouve plus que chez le Saumon de Loire. Pour l'essentiel de la population, la phase de grossissement du saumon de l'axe Loire-Allier se déroule à $5000 \mathrm{~km}$ de nos côtes dans le détroit de Davis, durant 2 à 3 ans pour plus de $90 \%$ des individus.

La population est ainsi constituée de poissons de taille exceptionnelle (atteignant jusqu'à $1,20 \mathrm{~m}$ pour plus de $10 \mathrm{~kg}$ ). Les premiers grands saumons d'hiver se présentent en estuaire dès l'automne pour se reproduire 1 an plus tard dans le Haut-Allier, après un parcours de 900 km jalonné d'obstacles durant lequel ils ne s'alimentent pas.

Cette souche est actuellement menacée d'extinction. La population du bassin de la Loire se chiffrait en dizaines de milliers d'individus au siècle dernier (de l'ordre de 40000 captures/an en Basse-Loire vers 1895), en milliers entre 1950 et 1975 ( 1500 à 2000 captures/an sur l'ensemble de l'axe restant fréquenté) et en dizaines depuis les années 1990 (pêche totalement interdite depuis 1994 pour sauvegarder la souche).

\subsection{La Truite de mer}

Très proche du saumon tant au plan de ses caractéristiques physiques que de son cycle biologique, la présence de la truite de mer est masquée par l'intérêt porté à l'autre grand salmonidé.

Historiquement celle-ci est moins bien représentée que le saumon dans le bassin de la Loire, mais elle offre d'intéressantes perspectives de développement dans la mesure où elle profite des actions menées en faveur du saumon et peut se contenter de zones de frai situées plus en aval, notamment dans le bassin de la Vienne. 


\subsection{Les Aloses}

Deux espèces d'aloses fréquentent le bassin de la Loire. L'Alose Feinte (Alosa fallax) représente moins de $5 \%$ des effectifs et se cantonne à la partie inférieure du réseau hydrographique jusqu'en Indre et Loire. Plus abondante, la Grande Alose (Alosa alosa) constitue l'essentiel des effectifs et tend à coloniser un plus vaste territoire. Sur le bassin de la Vienne, celle-ci reste bloquée à l'aval du barrage de Maisons Rouges où elle se reproduit avec l'Alose Feinte produisant des hybrides qui fragilisent le stock.

Historiquement cette espèce fréquentait la plus grande partie du cours de la Loire (jusqu'à hauteur du Puy-en-Velay) ainsi que ses affluents. Très sensible aux obstacles et à leurs effets cumulatifs, l'aire de répartition de la Grande Alose a été sensiblement réduite dès l'aménagement des premiers ouvrages de navigation à partir du début du XVIII ${ }^{\mathrm{ème}}$ siècle, avant les grands équipements hydroélectriques.

Grâce à sa forte fécondité, l'espèce se maintient dans la mesure où elle peut utiliser (dans des conditions limitantes) certaines frayères forcées à l'aval des obstacles. Épisodiquement, la population se développe à l'occasion des années de forte hydraulicité printanière qui lui permettent de franchir les premiers obstacles et de reconquérir une partie de ses zones naturelles de reproduction.

Cette sensibilité aux conditions de migration est exacerbée en raison des délais très courts imposés par la biologie de l'espèce. Quelques mois seulement séparent les pics de migration vers l'amont (mai-juin) de la dévalaison des premiers alosons (août-septembre).

\subsection{Les Lamproies}

En dépit de leur intérêt pour la pêche professionnelle, la biologie et la dynamique de population des lamproies sont très mal connues.

La Lamproie marine (Petromizon marinus) forme l'essentiel des populations du bassin de la Loire ; elle est bien répartie sur la plupart des axes de migration jusque sur les contreforts montagneux (bassin de la Vienne, Morvan, Allier). La Lamproie fluviatile (Lampetra fluviatilis) est seulement signalée de façon occasionnelle à l'aval du bassin.

La remontée des adultes s'étale sur une longue période de migration à partir de fin décembre en Basse-Loire, dans des conditions de débits favorables. L'espèce bénéficie également d'aptitudes particulières au franchissement des obstacles, combinant ses capacités de nage et d'adhérence aux parois.

Elle est réputée sensible à la qualité générale du milieu aquatique en raison de ses relations étroites avec le lit des rivières, durant sa longue phase de croissance juvénile (2 à 5 ans) enfouie dans les sédiments à l'aval des frayères.

C'est actuellement l'espèce migratrice qui résiste le mieux à l'état actuel du bassin de la Loire.

\subsection{L'Anguille}

L'Anguille représente, en valeur économique, plus des $3 / 4$ de la production des pêcheries professionnelles du bassin de la Loire.
Contrairement aux autres grands migrateurs, cette espèce ne remonte pas les cours d'eau pour se reproduire mais pour occuper ses zones privilégiées de croissance et de maturation, dans les parties continentales du bassin.

Après leur phase de migration active au stade civelle et anguillette, les anguilles sub-adultes progressent plus lentement vers l'amont, stimulées notamment par la densité de population. Elles diffusent ainsi à travers le tissu hydrographique saturant progressivement la capacité d'accueil du bassin par l'aval.

Son aire de répartition la conduit, par vagues de colonisation successives, des marais de l'Atlantique jusqu'aux ruisseaux de Lozère, aussi haut que la limite de remontée du Saumon.

C'est une mauvaise nageuse, mais elle dispose de plusieurs années pour effectuer son parcours migratoire et bénéficie de certaines aptitudes à ramper sur les pentes rugueuses des anciens barrages de moulins.

\section{LES OBJECTIFS DE RESTAURATION}

\subsection{Principes d'orientation}

Les objectifs de restauration des poissons migrateurs du bassin de la Loire tiennent compte de nombreux éléments de cadrage et de planification.

Ils portent tout d'abord sur des cours d'eau et des espèces qui bénéficient de statuts migratoires, notamment en vertu de l'article L 232.6 du Code Rural.

Pour tous les axes prioritaires figurant sur la carte d'objectif (voir ci-après), l'aménagement de dispositifs de franchissement s'impose aux maîtres d'ouvrage, non seulement sur les ouvrages nouveaux ou en phase de renouvellement de titre, mais aussi sur tous les ouvrages existants dans la mesure où la liste des espèces migratrices à prendre en compte est fixée par arrêté ministériel.

Dans ce cadre réglementaire, les objectifs prioritaires se limitent à la restauration des parcours où le rétablissement de la libre circulation est réaliste à moyen terme, c'est-à-dire jusqu'aux principaux verrous amont, à hauteur des grands ouvrages de fermeture des gorges (complexe hydro-électrique de I'lle Jourdain sur la Vienne, la Roche Bat l'Aigue - La Roche aux Moines - Eguzon sur la Creuse, QueuilleLes Fades sur la Sioule - Villerest-Grangent sur la Loire).

Même à l'intérieur de ces limites, les axes prioritaires offrent des marges de reconquête appréciables en terme de surface productive pour la fraie et le développement des poissons migrateurs.

Guidant le choix des axes prioritaires, ce potentiel productif accessible est d'autant plus important que les affluents développent de vastes capacités d'accueil et que la restauration des conditions de libre circulation s'accompagne d'améliorations dans la gestion des débits à l'aval des grands ouvrages.

La cohérence et la validité des objectifs prioritaires de restauration des poissons migrateurs sont assurées par la convergence de nombreux documents de cadrage et de pro- 
grammation. Ils figurent au niveau national dans le «Contrat Retour aux Sources» (1992) ; au niveau du bassin dans les décisions du Plan Loire Grandeur Nature (1994), dans le Schéma Directeur d'Aménagement et de Gestion des Eaux (1996) et le Plan de Gestion des Poissons Migrateurs (1996) ; plus localement dans les Schémas Départementaux de Vocation Piscicole, les contrats de rivières, ....

\subsection{Sauvegarder et réacclimater le Saumon}

A l'horizon 2005 les actions en faveur du Saumon visent le rétablissement d'une population viable sur l'Allier et la réintroduction de l'espèce sur cinq axes complémentaires pour atteindre la saturation de 500 ha de frayères (Allier, Sioule, Dore, Allagnon, Gartempe, Arroux).

Cet objectif correspond au retour d'environ 3000 adultes sur les frayères du bassin de la Loire après un prélèvement du même ordre de grandeur par la pêche.

\subsection{Reconquérir le potentiel productif des stocks d'Aloses, de Lamproies et de la Truite de mer}

Concernant l'Alose, les objectifs correspondent au rétablissement d'un niveau d'abondance en rapport avec les potentialités des milieux productifs existant sur les cours aval et médian des grands axes du bassin : Vienne, Creuse, Mayenne, Allier, Loire, Arroux. Les frayères, situées à l'aval des limites supérieures de migration, doivent être rendues accessibles et réactivées.

Compte tenu de la prolificité des aloses et de la courte durée de leur cycle biologique, on attend de cette espèce une réponse dynamique à la levée des blocages migratoires.

Pour la Lamproie, les objectifs sont plus difficiles à définir étant donné le manque de connaissance sur la dynamique des populations. Toutefois, compte tenu de la bonne répartition de l'espèce dans le bassin de la Loire, on peut escompter un renforcement des stocks à travers les actions générales menées sur l'ensemble du bassin en matière de restauration de la qualité des eaux et du réseau de migration.

De même, en dépit des incertitudes qui subsistent sur l'origine des populations de truites de mer, la réouverture de sous-bassins comme celui de la Vienne et les actions menées en faveur du saumon permettent d'espérer une augmentation des populations dans le bassin de la Loire.

\subsection{Enrayer la régression de la population d'anguilles}

Compte tenu du mode de renouvellement de la population d'Anguille, le recrutement en civelles au niveau de l'estuaire de la Loire ne dépend pas de la situation du stock à l'intérieur du bassin, mais de l'état général de la population à l'échelle européenne.

Pour autant, il est réaliste de se fixer un objectif portant sur l'état de la fraction de stock en phase de croissance et de maturation à l'intérieur du bassin. Il s'agit d'enrayer la régression de la colonisation du réseau hydrographique ligérien en intervenant sur les conditions de migration et sur la gestion des captures.
Plus précisément le but à moyen terme est de maintenir l'aire de répartition actuelle et la densité de population jusqu'aux premiers grands obstacles du réseau (ouvrages de plus de $10 \mathrm{~m}$ ). Cet objectif minimum implique d'ores et déjà la mise en place d'actions significatives dans les prochaines années.

\section{III 圆 LES FACTEURS LIMITANTS ET LES PROGRAMMES D'ACTIONS}

\subsection{Qualité de l'eau}

\section{- à l'intérieur du bassin}

Les programmes de l'Agence de l'Eau et de ses partenaires en matière de prévention et de lutte contre les pollutions commencent à donner des résultats significatifs dans le domaine de l'élimination des pollutions organiques ainsi que dans la réduction de la pollution par les phosphates. Cette évolution a des effets positifs sur la qualité des zones de reproduction et de développement des poissons migrateurs. Elle renforce les perspectives de reconquête des capacités d'accueil et de production, notamment dans les secteurs concernés par les Plans d'Actions Renforcés et les contrats de rivières (Vienne, Cher, Dore, ...).

\section{- dans l'estuaire}

Au plan de la dégradation de la qualité des eaux, un point sensible et le plus limitant pour les espèces amphihalines est l'exutoire du bassin versant.

Au regard de sa situation trophique et géomorphologique, l'estuaire de la Loire est devenu particulièrement vulnérable à l'impact du bouchon vaseux. Dans le cas de la Loire, la dynamique du phénomène est liée à l'importance du bassin d'alimentation (120000 km²), la réduction des surfaces d'auto-épuration disponibles dans l'estuaire (vasière, bras secondaires, zones d'expansion), la remontée du bouchon vaseux dans la partie amont de l'estuaire sous l'effet de l'abaissement de la ligne d'eau et la charge organique du fleuve en périodes d'eutrophisation.

Le processus de décomposition et de désoxygénation qui en découle (mort et dégradation des algues d'eau douce en milieu saumâtre turbide) est d'autant plus intense que l'état d'eutrophisation du fleuve est important, que la température de l'eau est élevée et que l'amplitude des marées favorise la remise en suspension de la crème de vase. Il atteint son maximum en période d'étiage et de vives eaux.

En année hydrologique normale le phénomène ne se manifeste pas avant le mois de juin et ne se prolonge pas après le mois de septembre (teneur en oxygène $\geq 6 \mathrm{mg} / \mathrm{l}$ ). Ainsi, eu égard aux périodes de migration, les poissons les plus vulnérables sont les premiers alosons qui dévalent à partir de la fin de l'été (dès le mois d'août) et les derniers saumoneaux dont la dévalaison s'étale jusqu'en juin. Les autres flux migratoires (grands saumons d'hiver et de printemps, lamproies adultes et juvéniles, aloses adultes, anguilles d'avalaisons, civelles) traversent l'estuaire sans être confrontés à l'effet annoxique du bouchon vaseux, dans la mesure où l'essentiel des passages a lieu en dehors de la 
période critique d'étiage, généralement à la faveur de fortes hydraulicités (températures peu élevées, débits favorables à la dilution et à l'expulsion du bouchon vaseux).

Cette fenêtre physico-chimique, laisse donc passer la plus grande partie des flux migratoires en année humide et normale, mais elle se réduit en situation d'étiage précoce ou prolongée, de telle sorte qu'en cas de sécheresses répétées (entre 1988 et 1992) l'écran biologique du bouchon vaseux peut mettre à mal des espèces sensibles et en sous-effectif.

Le contrôle de l'extension du phénomène et sa résorption passent par un ensemble d'actions complémentaires, menées à long terme, à l'échelle de l'estuaire et du bassin versant. Cette reconquête de la qualité générale du milieu est engagée à travers une série d'actions parmi lesquelles on peut citer : - la priorité donnée à la lutte contre l'eutrophisation dans le cadre des programmes de l'Agence de l'eau Loire-Bretagne,

- le programme d'assainissement de l'agglomération nantaise,

- la prise en compte des pollutions d'origine agricole,

- l'élargissement des moyens de prévention à l'entretien des fonctions auto-épuratrices des cours d'eau et à la mise en place des bandes enherbées,

- l'arrêt des extractions de granulats en lit mineur.

- la modélisation prospective de l'estuaire et la priorité donnée au relèvement de la ligne d'eau d'étiage,

- la protection des zones humides,

- la réhabilitation de bras secondaires, etc....

\subsection{Prélèvements par la pêche}

Depuis 1995, le calage des mesures réglementaires concernant la pêche des poissons migrateurs (ouvertures, engins, quotas, ...) et le suivi de la ressource s'inscrivent dans un cadre de gestion unique et décentralisé à l'échelle du bassin versant (décret $\mathrm{n}^{\circ}$ 94-157 du 16 février 1994). A cet effet, un plan de gestion est établi par le Comité de Gestion des Poissons Migrateurs du bassin de la Loire (COGEPOMI) regroupant des représentants des services de l'État et de ses établissements publics, des pêcheurs de loisirs, des pêcheurs professionnels fluviaux, des marins pêcheurs exerçant en estuaire, des propriétaires riverains et des collectivités territoriales du bassin. Cette instance présidée par le Préfet de la Région des Pays de la Loire est chargée d'évaluer et de rechercher les règles optimales d'exploitation des ressources amphihalines dans le respect du patrimoine biologique et des réglementations en vigueur.

La pêche du saumon est ainsi totalement interdite en amont de la limite de la mer (dans le domaine de compétence du Comité de Gestion des Poissons Migrateurs) pendant la durée du plan de gestion 1995-1999.

En outre, sur les zones de grossissement en mer, les pêches au large des Iles Féroé sont interdites depuis 1992 (rachat des quotas de pêche par le North Atlantique Salmon Found) et les pêcheries à l'ouest du Groënland sont arrêtées depuis 1993 à la suite d'un moratoire qui pourrait être suivi par un rachat de quotas.

\subsection{Qualité des habitats aquatiques}

Les migrateurs qui se reproduisent en eau douce le font principalement dans des secteurs hydrographiques où l'habitat aquatique est le mieux préservé de la pression anthropique. Dans ces conditions, la qualité du milieu physique ne constitue pas actuellement un facteur limitant pour les populations amphihalines du bassin. Néanmoins, l'arrêt des extractions de granulats en lit mineur, la réduction des travaux de recalibrage en zone rurale, la réhabilitation des ripisylves et la généralisation des techniques douces d'entretien des capacités d'écoulement constituent un changement de politique d'intervention favorable au fonctionnement des cours d'eau à migrateurs (restauration du lit de la Loire, Gartempe, Arroux, Dore, Sioule, ...).

Plus directement étudiée en fonction des besoins hydrobiologiques et piscicoles, la gestion des débits réservés et des lâchures, la réduction de l'impact des éclusées et des vidanges de barrage, constituent une voie d'amélioration importante des conditions de reproduction et de développement sur le cours amont des parcours de migration ; ce type d'actions est en cours, notamment dans le bassin de l'Allier.

\section{공 Circulation piscicole}

La principale cause de régression des poissons migrateurs du bassin de la Loire est l'édification des barrages. Inversement, la restauration des circuits de migration constitue au même titre le principal domaine d'intervention spécifique pour la reconstitution des stocks.

\section{— bassin de la Maine}

Toutes les caractéristiques hydrographiques du bassin de la Maine en font un territoire prédestiné à l'accueil et au développement de l'Anguille : sa proximité océanique (60 $\mathrm{km}$ de l'estuaire), sa surface colonisable (plus de 1/5ème du bassin de la Loire sans entrave par les grands barrages) et sa faible altitude.

La réhabilitation de ce réseau migratoire équilibre le programme général au plan de la répartition géographique (région aval) et au plan spécifique (prise en compte de l'Anguille).

L'opération débute par une étude de potentialité et de définition des actions prioritaires. Une première tranche d'intervention vise la réouverture de l'axe Mayenne qui bénéficie d'un statut migratoire privilégié et de potentialité complémentaire facilement accessible en faveur de l'Alose.

\section{- bassin de la Vienne}

Le réseau hydrographique de la Vienne est un territoire fondamental pour la restauration des poissons migrateurs du bassin de la Loire. Il fait partie des sous-bassins les plus vastes et les plus proches de l'estuaire.

De plus, la façade atlantique des contreforts du Massif Central offre des milieux d'excellente qualité, intéressant toutes les espèces concernées par le programme.

L'exploitation de ce potentiel est bloquée par le barrage de Maisons Rouges très mal situé à la base du réseau, juste à l'aval du confluent entre la Vienne et la Creuse. Ce verrou qui, en dépit des réaménagements de son dispositif de franchissement obère, entre autres, les résultats du programme 


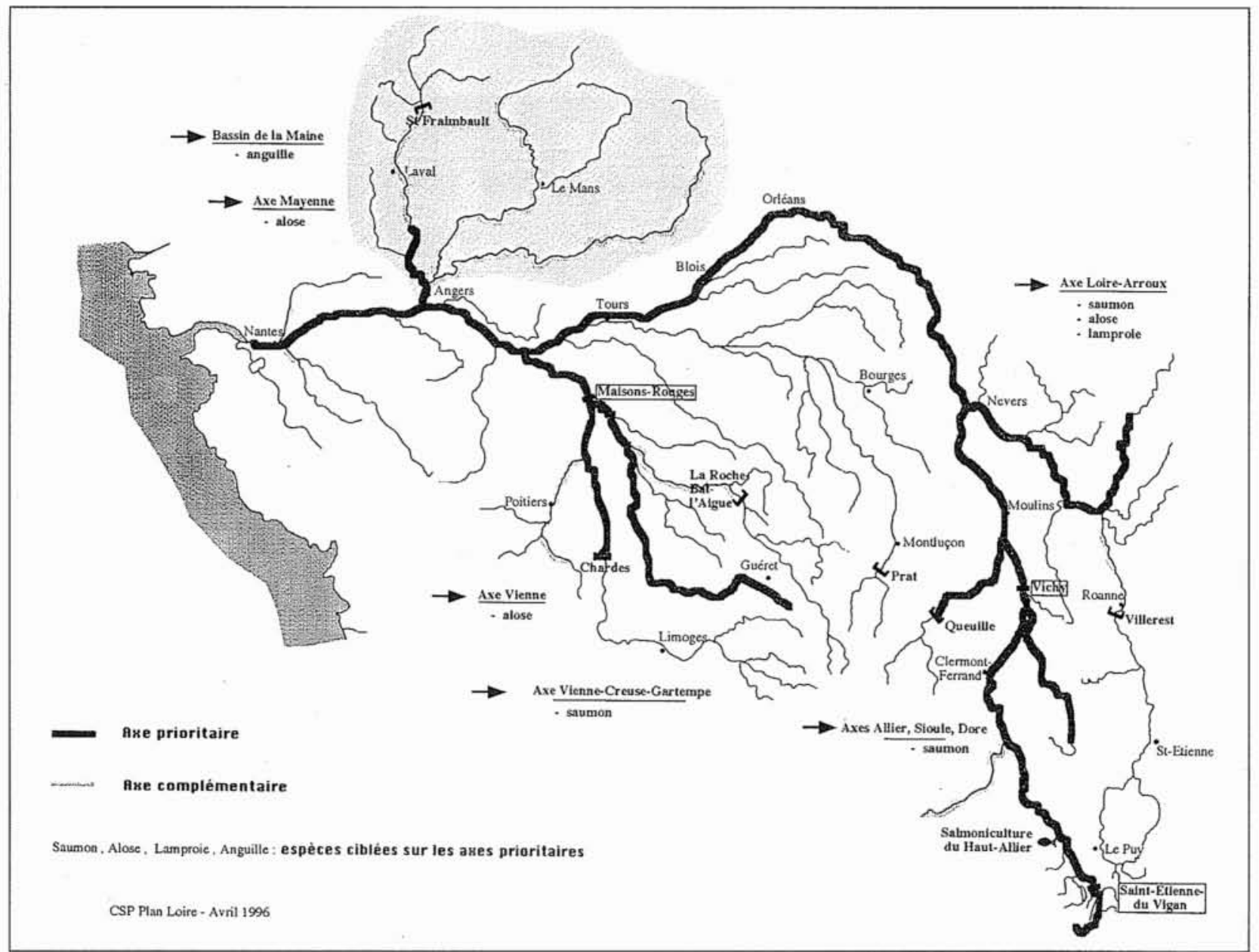

\section{Restauration des poissons migrateurs du bassin de la Loire.}

de restauration de l'axe Creuse-Gartempe pour le Saumon, fait l'objet d'une décision d'effacement dans le cadre du Plan Loire Grandeur Nature.

En complément de cette action décisive, des aménagements de passes à poissons doivent être entrepris pour optimiser la restauration de l'axe Gartempe et mettre en valeur les potentialités des deux axes principaux, la Vienne et la Creuse en faveur de l'Alose, la Lamproie, la Truite de mer et l'Anguille.

\section{- bassin du Cher}

Compte tenu de sa dimension, de sa position relativement proche de l'estuaire et de sa typologie de bassin de plaine, le réseau hydrographique du Cher offre d'importantes capacités d'accueil pour l'Anguille. Il reste en outre fréquenté de façon relictuelle par la Lamproie, l'Alose et la Truite de mer malgré la présence d'un barrage très difficilement franchissable à Tours sur son cours aval.

Le Cher ne figure pas sur la liste des axes prioritaires du "Contrat Retour aux Sources». Toutefois, la mise en conformité du barrage de Tours et la prise en compte des besoins de fluidité migratoire dans le cadre de la modernisation des ouvrages du Cher canalisé devraient assurer la reconstitution des stocks d'anguilles, de lamproies et d'aloses sur cette branche importante du réseau ligérien.

\section{- Loire moyenne}

De la confluence du Cher à celle de l'Allier, la Loire moyenne est un long couloir de migration $(300 \mathrm{~km})$ sans aucun affluent important susceptible d'élargir les potentialités d'accueil des poissons migrateurs. La qualité de ce tronçon s'est dégradée depuis la fin des années 60 par l'aménagement ou le renforcement de seuils (centrales nucléaires, radiers de pont) et la déstabilisation consécutive aux extractions de granulats. La restauration de la fluidité migratoire de cet axe majeur est un élément important pour la mise en valeur des bassins amont, compte tenu de l'impact cumulatif des obstacles sur de tels parcours. Elle est engagée dans le cadre de la restauration du lit de la Loire et du renouvellement des titres de concession des ouvrages E.D.F.

\section{- l'axe Loire-Arroux}

Le réseau du Morvan constitue la partie du bassin de la Loire la mieux conservée au plan de la libre circulation piscicole. Les aménagements des dispositifs de franchissement 
ou améliorations qui restent à apporter se limitent à trois ouvrages. Ces interventions ponctuelles permettront de réhabiliter $300 \mathrm{~km}$ d'axes migratoires vers lesquels s'engagent encore d'importants flux d'aloses, de lamproies et d'anguilles, auxquels il faut ajouter les perspectives de réintroduction du saumon.

\section{— bassin de l'Allier}

La plupart des obstacles construits dans le lit de l'Allier ou apparus sous les effets des extractions de granulats ont été équipés de passes à poissons depuis l'engagement du $1^{\text {er }}$ plan-saumon en 1976. Parmi les plus grosses difficultés migratoires, le barrage de Poutès a été équipé d'un ascenseur : plus récemment le dispositif de franchissement du pont-barrage de Vichy a été réaménagé pour le Saumon mais aussi pour l'Alose dans le cadre du Plan Loire ; enfin, l'effacement du barrage de Saint-Etienne-du-Vigan est prévu dans ce même cadre de programmation pour obtenir la reconquête intégrale de l'axe.

En ce qui concerne les axes secondaires, la plus grande partie des obstacles a été ou est en voie d'équipement, grâce aux contrats de rivière : aménagements réalisés sur la Sioule, en cours sur la Dore, en projet sur l'Allagnon.

Il reste néanmoins des améliorations et des équipements complémentaires à réaliser pour parfaire la restauration du réseau de migration du saumon dans le bassin de l'Allier.

\subsection{Repeuplement}

Les actions de repeuplement concernent le Saumon. Elles sont partie intégrante du programme de restauration.

La réalisation de la salmoniculture du Haut-Allier a été décidée avec la mise en place du Plan Loire pour sauvegarder la souche du Saumon de l'Allier et ramener en l'espace de 10 ans la population ligérienne à un niveau d'abondance et de distribution géographique en rapport avec les capacités migratoires restaurées dans le bassin de la Loire.

Pour atteindre les objectifs fixés à partir de la capacité d'accueil des frayères accessibles ou en cours de reconquête (500 ha) le dispositif devra produire 500000 oeufs en incubateurs de terrain, 1000000 de tacons de moins de 3 mois et 300000 saumoneaux âgés d'un an.

Cet effort de relance est justifié par l'importance des embûches qui jalonnent les parcours de migration et par la faiblesse du stock relictuel. En outre, le réensemencement des affluents où le Saumon a disparu, exige des alevinages massifs pour augmenter les chances de réussite des opérations de réintroduction.

L'état de précarité de la population qui subsiste sur l'Allier nécessite la mise en oeuvre de moyens techniques sûrs et performants, avec un prélèvement de géniteurs sauvages limité au strict minimum, sans dégradation de l'intégrité génétique du stock.

Le reconditionnement des géniteurs sauvages (pour une reproduction en pisciculture plusieurs années successives), le recours à une production intermédiaire de géniteurs enfermés, l'adaptation de l'élevage des juvéniles au mode de croissance élevé des saumoneaux sauvages, la limitation des risques sani- taires et génétiques, sont autant de contraintes et d'exigences de qualité qui ont conduit au choix d'une solution technique basée sur une parfaite maîtrise des paramètres d'élevage.

\subsection{Suivi des stocks et évaluation des résultats}

L'évaluation des résultats fait partie intégrante du programme de restauration des poissons migrateurs du bassin.

Le suivi des retours est effectué au moyen de stations de contrôle installées en différents points des circuits de migration au niveau des passes à poissons. Il en existe deux sur l'axe Allier (Vichy et Poutès). Une est en projet à l'entrée du Morvan sur l'axe Loire-Arroux (Decize).

Pour compléter ce réseau, il est envisagé d'implanter d'autres stations, notamment sur la Sioule, sur l'Allier à l'entrée de la zone des meilleures frayères à saumons, sur l'Arroux et sur les axes du bassin de la Vienne.

Le suivi des migrations porte également sur le comptage des points de frai (nids de ponte pour le Saumon), sur l'évaluation des densités de juvéniles en phase de croissance dans les zones de production (pêche électrique sur les radiers à tacons), et sur le contrôle des dispositifs de dévalaison (exutoire de Poutès).

Le suivi chronologique des captures réalisées par les pêcheries, leur validation par réseau de pêcheurs échantillonneurs et leur transcription en terme de captures par unité d'effort fournissent également des données utiles à la gestion des stocks et à l'évaluation des programmes de restauration. Un indice d'abondance a été mis au point à cet effet par l'Association Agréée Interdépartementale des Pêcheurs Professionnels du Bassin de la Loire et des Cours d'Eau Bretons.

\section{IV $\mathbf{a}$ CONCLUSION}

Si les poissons migrateurs du bassin de la Loire sont des espèces passionnantes en elles-mêmes du fait de leur cycle biologique qui les conduit à parcourir de très grandes distances pour venir se reproduire dans les rivières qui les ont vu naître, ils ont également un intérêt économique et une valeur patrimoniale d'ensemble significative au niveau d'un bassin.

L'extinction progressive des différentes espèces de poissons migrateurs de ce bassin ne pourrait être que le révélateur d'une situation extrêmement préoccupante devant conduire à se poser des questions fondamentales sur les actions de l'homme sur l'environnement et le milieu aquatique.

A l'inverse lorsque l'homme se fixe des objectifs ambitieux de restauration du milieu aquatique, il est très vite conduit à chercher un indicateur tangible et perceptible des progrès réalisés. Il peut alors se tourner vers les poissons migrateurs : c'est l'exemple de la réintroduction du saumon dans le Rhin qui commence à porter ses premiers fruits.

Souhaitons que pour le bassin de la Loire les efforts et la raison de tous permettent de conserver et de restaurer ce patrimoine inestimable que sont les poissons migrateurs sans attendre la disparition de certaines espèces pour prendre conscience de cette richesse. 